\title{
Leonardo knew the fluid boundaries of science
}

\section{Without the aid of mathematical models, his ideas stemmed from keen observation.}

Sir - The Science in Culture article "Leonardo lifts off" (Nature 421, 792; 2003) provided a critique of the myth that Leonardo da Vinci was "a man ahead of his time", by suggesting that his plans for a successful flying machine design depended more on luck than his knowledge of fluid dynamics. In particular, it was stated that Leonardo "did not pay attention to the fact that air, unlike water, is compressible, and had not considered such a possibility".

I believe that a number of writings by Leonardo indicate the contrary. On a page now in the Codice Atlantico (at the Ambrosiana Library in Milan), Leonardo wrote: "Water is incompressible. ... The opposite is the case for air, which when forced into vases with small openings, containing some water, ... drives away the water with such fury (furore) that it will be sprayed a great distance away, till that air that remains in the vase recover its initial density" (the translation from the Italian original is mine).

In the same collection there are notes made by Leonardo about the compression

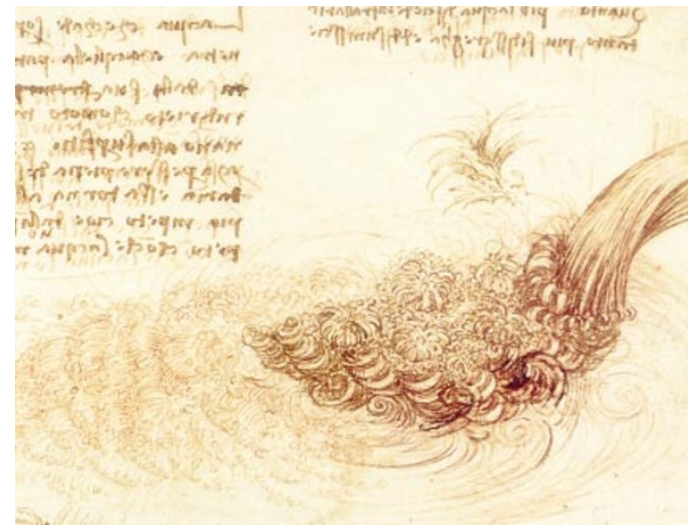

Data stream: Leonardo's Study of Flowing Water.

of air below birds' wings during flight.

I do not think, however, that the correctness of observations such as these makes Leonardo any closer to contemporary science. Leonardo, unlike Galileo and Newton, did not rely on abstract mathematical models, capable of producing quantitative predictions, which could then be confirmed by experiment. His own studies in fluid dynamics show this very clearly. They were firmly rooted in extremely penetrating visual observation, as witnessed by his drawings of water streams (see figure), but they totally lacked the mathematical approach of Bernoulli's Hydrodynamica (1738).

In Leonardo's water studies, the separation between art and science itself becomes a fluid boundary. His Study of Flowing Water, made in about 1509-1511, is a masterly representation of water flow carried out with supreme keenness of observation and attention to detail, powerfully conveying the sense of movement of the fluid. Nature here is not yet understood in terms of abstract concepts and mathematical models; rather, its phenomena (and movement in particular) are directly grasped by the mind through the eye, in a manner which is closer to our experience of aesthetic contemplation than to the approach of modern science.

Stefano E. Grillo

Institute of Materials Science and Process Engineering (CNRS), Tecnosud,

Université de Perpignan, 66100 Perpignan, France

\section{Open access: other ways}

Sir — John Ewing, in Correspondence, argues that open-access journals are not open to everyone because not all authors can pay or find a sponsor to pay their processing fees (Nature 425, 559; 2003). Although publishers of open-access journals such as the Public Library of Science (PloS) say that authors who can't pay won't have to, Ewing feels they have underestimated the numbers who will not be able to pay.

As a long-time advocate of open access to science, I can list several points to suggest that this concern is overstated.

Declan Butler notes in his News Feature "Scientific publishing: who will pay for open access?” (Nature 425, 554-555; 2003) that many funding organizations are willing to pay these fees for their grant recipients. Although this solution will not work in disciplines that are less well-funded than medicine (in Ewing's field of mathematics, for example, or mine, philosophy), that is no objection to applying it to fields where it can work. It is highly likely, and desirable, that different fields will develop different open-access models. Some peerreviewed open-access journals in the humanities charge no processing fees at all.

Ewing notes that many universities may not be able to afford these fees, in cases when funding bodies cannot pay them. But if open access spreads, every university will make large savings from the conversion, cancellation or demise of expensive subscription-based journals. This money can be used to support the open-access model of archiving and publication.

If some future open-access publishers have no policy to waive fees, and authors find themselves excluded on financial grounds alone, there are other ways to bring about open access to peer-reviewed literature (such as e-print archiving) that do not depend on open-access journals, research grants, affluent employers or windfall savings in the library budget. Peter Suber

Department of Philosophy, Earlham College, Richmond, Indiana 47374-4095, USA; Public Knowledge, 1875 Connecticut Avenue, NW, Washington DC 20009, USA

\section{Open access will deter illegal file-sharing}

Sir — John Ewing argues in Correspondence (Nature 425, 559; 2003) that, in the current reader-pays publishing system, researchers without a journal subscription can obtain an article by other means. This is probably true. However, article-sharing is an illegal and unreliable method of getting scientific information, because many commercial publishers own copyright as well as the rights to distribute the results. The magnitude of this copying activity is, as far as I know, unknown.

A few requests to "send me a PDF file of an article" will probably not hurt the current system. However, if this approach were to become more systematic, a PDF file (or other formats) could be 'shared' using databases and peer-to-peer networks just as any MP3 music file can be shared worldwide, to the great annoyance of the music industry. This would definitely hurt the current subscriber-pays system.

The open-access alternative is immune to such copying activities because the articles are available free of charge. Martin Dufva

MIC-Mikroelektronik Centret, Oersteds Plads, Technical University of Denmark, Building 345 East, DK-2800 Kgs. Lyngby, Denmark

The Nature Publishing Group does not retain copyright. Authors grant NPG an exclusive licence; in return they are free to reuse their papers in their future printed work, and to post a copy on their own notfor-profit website. See http://npg.nature. com/authornews - Editor, Nature. 